\title{
Downregulation of FOXP2 promotes breast cancer migration and invasion through TGF//SMAD signaling pathway
}

\author{
MENG-TING CHEN ${ }^{1,2^{*}}$, HE-FEN SUN ${ }^{1,2^{*}}$, LIANG-DONG LI ${ }^{1,2^{*}}$, \\ YANG ZHAO $^{1,2}$, LI-PENG YANG ${ }^{3}$, SHUI-PING GAO ${ }^{1,2}$ and WEI JIN ${ }^{1,2}$ \\ ${ }^{1}$ Department of Breast Surgery, Key Laboratory of Breast Cancer in Shanghai, \\ Collaborative Innovation Center of Cancer Medicine, Shanghai Cancer Center, Fudan University; \\ ${ }^{2}$ Department of Oncology, Shanghai Medical College, Fudan University; ${ }^{3}$ Department of Pathology, \\ School of Basic Medical Sciences, Fudan University, Shanghai 200030, P.R. China
}

Received November 29, 2017; Accepted March 19, 2018

DOI: $10.3892 / \mathrm{ol} .2018 .8402$

\begin{abstract}
Cancer metastasis and relapse are the primary cause of mortality for patients with breast cancer. The present study performed quantitative proteomic analysis on the differentially expressed proteins between highly metastatic breast cancer cells and parental cells. It was revealed that forkhead box P2 (FOXP2), a transcription factor in neural development, may become a potential inhibitor of breast cancer metastasis. The results demonstrated that patients with a lower level of FOXP2 expression had significantly poorer relapse-free survival $(\mathrm{P}=0.0047)$. The transcription of FOXP2 was also significantly downregulated in breast cancer tissue compared with normal breast tissue $(\mathrm{P}=0.0005)$. In addition, FOXP2 may inhibit breast cancer cell migration and invasion in vitro. It was also revealed that the underlying mechanism may include the epithelial-mesenchymal transition process driven by the tumor growth factor $\beta / \mathrm{SMAD}$ signaling pathway. In conclusion, the present study identified FOXP2 as a novel suppressor and prognostic marker of breast cancer metastasis. These results may provide further insight into breast cancer prevention and the development of novel treatments.
\end{abstract}

\section{Introduction}

Breast cancer remains one of the most prevalent types of cancer among women. It is estimated that there will be

Correspondence to: Professor Wei Jin, Department of Breast Surgery, Key Laboratory of Breast Cancer in Shanghai, Collaborative Innovation Center of Cancer Medicine, Shanghai Cancer Center, Fudan University, 270 Dong-An Road, Shanghai 200030, P.R. China E-mail: jinwei7207@163.com

*Contributed equally

Key words: breast cancer metastasis, forkhead box P2, transcription factor, transforming growth factor $\beta / \mathrm{SMAD}$, epithelial-to-mesenchymal transition
255,180 new breast cancer cases and 41,070 deaths in the U.S. in 2017 (1). Most of the mortality is caused by cancer invasion and metastasis. However, there are still limited effective therapies for metastatic breast cancer patients. In our previous study, we investigated the differentially expressed proteins between parental MDA-MB-231 and highly metastatic MDA-MB-231 (MDA-MB-231HM and MDA-MB-231BO) breast cancer cell lines (2-4). We found that forkhead box P2 (FOXP2), a transcription factor, had a significantly reduced expression level in highly metastatic cell lines. FOXP2 is a member of FOXP gene subfamily (FOXP1, FOXP2, FOXP3, FOXP4) which all have a C-terminal winged helix forkhead DNA binding domain. The structure is conserved in many species. It was previously proved that the gene FOXP2, locating at 7q31, regulates many neurogenesis signaling pathways critical in embryonic development and cell cycle such as Hedgehog, WNT and Notch pathways $(5,6)$. It is also involved in lung, heart and central nervous system (CNS) development. Especially, it plays pivotal roles in human language development. Depletion and mutation in FOXP2 can lead to speech and linguistic impairment, aging and cancer $(7,8)$.

Several researches have also reported the roles of FOXP2 as a tumor suppressor in gastric cancer (9), osteosarcoma (10) and hepatocellular carcinoma (11) previously. It was revealed that the expression of FOXP2 was usually regulated by a series of microRNAs in cancer cells. However, there were not many studies investigating the mechanisms of FOXP2 in breast cancer. In our current study, we explored the effects and mechanisms of FOXP2 on breast cancer metastasis and prognosis. We hope our study could provide deeper insights into the diagnosis and treatment of metastatic breast cancer.

\section{Materials and methods}

Tumor samples. Our study has already been approved by the Ethical Committee and Institutional Review Board of Fudan University Shanghai Cancer Centre (FDUSCC). The methods were performed in accordance with the approved guidelines. All the participants signed written informed consent forms. The tumour samples were collected between 
May 2014 and May 2015. The breast cancer samples and matched para-carcinoma samples were collected from 39 patients pathologically diagnosed with breast cancer who undergone surgery. The samples were frozen immediately in liquid nitrogen after surgery.

Reverse transcription-quantitative polymerase chain reaction $(R T-q P C R)$. Total RNA was extracted using TRIzol reagent (Invitrogen; Thermo Fisher Scientific, Inc., Waltham, MA, USA) and reverse transcribed with PrimeScript RT Reagent kit (Takara Biotechnology Co., Ltd., Dalian, China). Subsequently, RT-qPCR was performed with SYBR Premix Ex Taq (Takara Biotechnology Co., Ltd.) using ABI Prism 7900 instrument (Applied Biosystems; Thermo Fisher Scientific, Inc.) according to the manufacture's protocol. The thermocycling condition was pre-denaturation at $95^{\circ} \mathrm{C}$ for $30 \mathrm{sec}$, denaturation at $95^{\circ} \mathrm{C}$ for $5 \mathrm{sec}$, annealing at $60^{\circ} \mathrm{C}$ for $30 \mathrm{sec}$, with 40 cycles. The relative gene expression was calculated using the $2^{-\Delta \Delta \mathrm{Cq}}$ method (12). The primer sequences used in this study are as follows: FOXP2: forward 5'-AAC AACAGCAGGCTCTCCAG-3', and reverse 5'-GGCACCTGC AGTGGTCTC-3'; GAPDH: forward 5'-GGTGGTCTCCTC TGACTTCAACA-3', and reverse 5'-GTTGCTGTAGCCAAA TTCGTTGT-3'.

Cell culture. The cells in this study were obtained from the Shanghai Cell Bank, Type Culture Collection Committee of Chinese Academy of Science (Shanghai, China). The cells for experiments were passaged for less than 6 months. MDA-MB-231, MDA-MB-231BO, MDA-MB-231HM and MDA-MB-468 (all triple negative breast cancer) cells were grown in Leibovitz L-15 medium (BasalMedia, Shanghai, China). 293T, MCF7 (luminal positive breast cancer) and BT549 (triple-negative breast cancer) cells were cultured in high-glucose Dulbecco's modified Eagle's medium (DMEM; HyClone; GE Healthcare Life Sciences, Logan, UT, USA). SKBR3 (HER2 positive breast cancer), T47D, ZR7530 and ZR75-1 (all luminal positive breast cancer) were maintained in RMPI-1640 medium (HyClone; GE Healthcare Life Sciences). All the medium was supplemented with $10 \% \mathrm{FBS}, 100 \mathrm{IU} / \mathrm{ml}$ penicillin, and $100 \mathrm{mg} / \mathrm{ml}$ streptomycin. All the cells were maintained with $5 \% \mathrm{CO}_{2}$ at $37^{\circ} \mathrm{C}$. The MDA-MB-231HM was developed from the parental MDA-MB-231 cell line via the tail vein in mice for four cycles. We have patent application for the cell line (patent no. 200910174455.4) which exhibited increased lung metastasis compared to parental cells. The MDA-MB-231BO cells, which have highly metastatic potential to bone, was obtained from Dr Toshiyuki Yoneda (University of Texas, Houston, TX, USA). We have done several studies with these cell lines $(4,13)$.

Plasmids and lentivirus packaging. Human FOXP2 cDNA was purchased from fulenGen and then subcloned into the pCDH-CMV-MCS-EF1-Puro lentiviral vector. The cloned primer sequence is as follows: FOXP2: forward 5'-CCG GAATTCGCCACCATGATGCAGGAATCTGCGAC-3', and reverse 5'-CGCGGATCCTCATTCCAGATCTTCAGATA-3'.

FOXP2 shRNAs and the negative control were purchased from GeneChem and expressed in the GV248 backbone. The target sequences are as follows: shRNA NC 5'-TTCTCCGAA CGTGTCACGT-3'; shRNA1: 5'-TTAACAATGAACACG CATT-3'; shRNA2: 5'-AGCAAACAAGTGGATTGAA-3'.

293 T cells were cotransfected with lentiviral vectors and the packaging vectors pCDH (GV248), psPAX2 and pMD2G. Virus-containing medium was collected $48 \mathrm{~h}$ after the transfection and added to the cancer cells.

Kinetic wound-healing assay. MDA-MB-231 and MDA-MB-231BO cells $\left(3.6 \times 10^{4}\right)$ were plated on 96-well plates (Essen ImageLock; Essen Biosciences, Ann Arbor, MI, USA), and a wound was scratched with wound scratcher (Essen Instruments). Wound confluence was monitored with Live-Cell Imaging System and software (Essen Biosciences). The wound closure was observed after $28 \mathrm{~h}$ by comparing the mean relative wound density of at least three biological replicates in each experiment.

Transwell assay. Cell migration and invasion was examined with Transwell chambers (BD Biosciences, Franklin Lakes, NJ, USA). The cells were seeded in the upper chamber in serum-free medium. The cell density was $5 \times 10^{5}$ for migration assay and $1 \times 10^{6}$ for Matrigel-coated invasion assay. Medium supplemented with $20 \%$ serum was added in the lower chamber. The cells were incubated for 15 to $20 \mathrm{~h}$. After that, the cells remained on the upper chamber were removed with cotton swabs. The cells on the lower surface of the membrane were stained with $0.4 \%$ methanol and $0.25 \%$ crystal violet.

Western blot analysis. Whole cell extracts were isolated with Pierce T-PER (Tissue Protein Extraction Reagent; Thermo Fisher Scientific, Inc.) containing protease inhibitor cocktail tablets (Roche Diagnostics, Indianapolis, IN, USA) and phosphatase inhibitors (Roche Diagnostics). Proteins $(30 \mu \mathrm{g})$ were resolved by sodium dodecyl sulphate-polyacrylamide gel electrophoresis (SDS-PAGE) and transferred onto a polyvinylidene fluoride membrane (Pall Corporation, Port Washington, NY, USA). The membranes were blocked in 5\% BSA and incubated in various primary antibodies followed by the corresponding horseradish peroxidase-conjugated secondary antibodies (Proteintech Group, Inc., Chicago, IL, USA). The primary antibody against FOXP2 (21608) and vimentin (40477) was obtained from Santa Cruz Biotechnology, Inc. (Dallas, TX, USA), fibronectin (66042-1-Ig) and GAPDH (60004-1-Ig) from Proteintech Group, Inc., N-cadherin (610920) from BD Biosciences, p-SMAD3 (ab52903) from Abcam (Cambridge, UK), SMAD3 (1735-s) from Epitomics (Burlingame, CA, USA), SMAD4 (9515p) from Cell Signaling Technology, Inc. (Danvers, MA, USA) and TGFßR1 (sc-398) from Santa Cruz Biotechnology, Inc. Antibody binding was detected using chemiluminescence (Amersham Imager 600; GE Healthcare Life Sciences), according to the manufacturer's instructions.

Statistical analysis. SPSS 22.0 software (IBM Corp., Armonk, NY, USA) was used to conduct the statistical analysis. Data were expressed as means \pm standard deviation. The student's t-test was used to evaluate the differences between two groups and one-way analysis of variance (ANOVA) was used among 
A

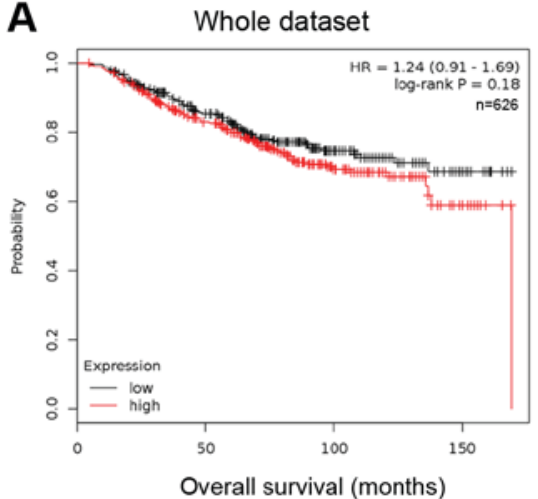

D

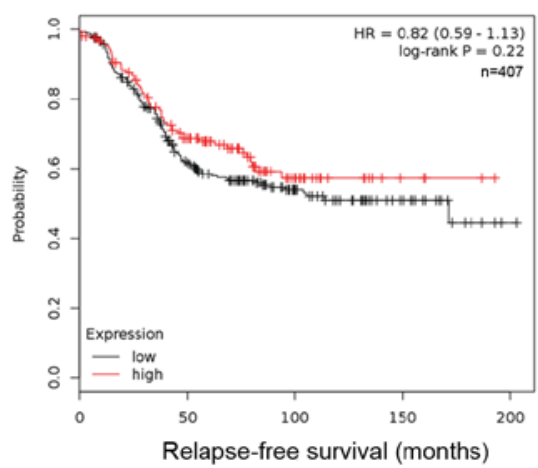

B

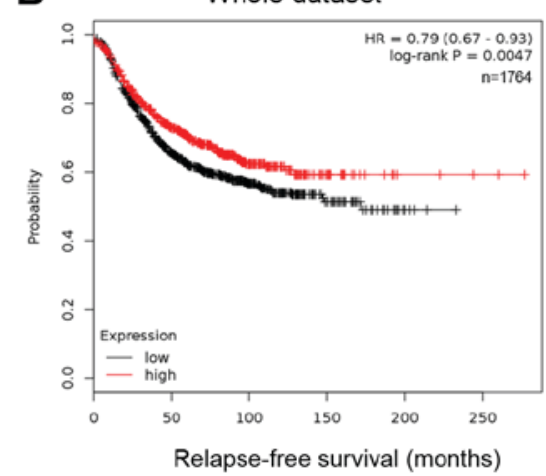

E

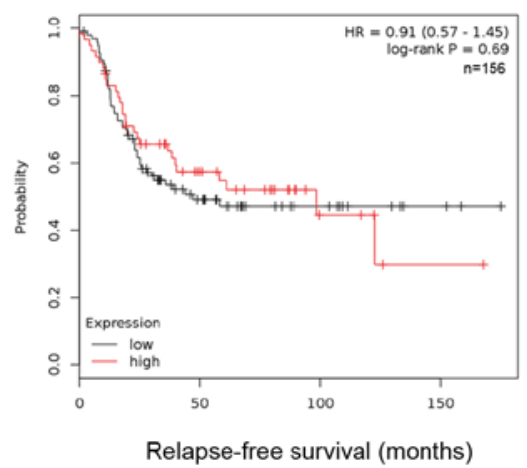

C

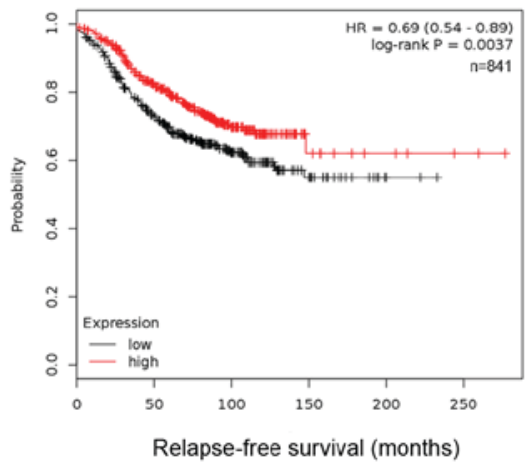

F

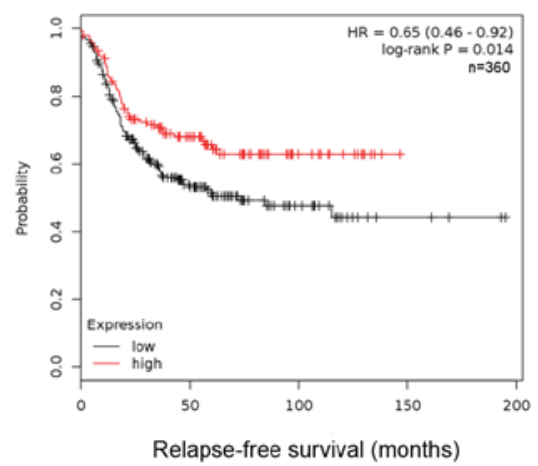

Figure 1. Lower FOXP2 expression predicts a shorter relapse-free survival in patients with breast cancer. Kaplan Meier (A) overall survival and (B) relapse free survival analysis of FOXP2 expression among patients of the whole dataset. Kaplan Meier relapse free survival analysis of FOXP2 expression among patients from the (C) Luminal A, (D) Luminal B, (E) HER-2 and (F) Basal-like subtypes from the kmplot website (kmplot.com/analysis/). HER-2, human epidermal growth factor receptor 2 .

multiple groups. LSD test was used after ANOVA to compare the differences between two groups. $\mathrm{P}<0.05$ was considered to indicate a statistically significant difference. Graphs were created with GraphPad Prism 5 (GraphPad Software, Inc., La Jolla, CA, USA).

\section{Results}

Low expression of FOXP2 indicated poorer prognosis in breast cancer. The information of FOXP2 expression and patients' survival was acquired from an online Kaplan-Meier plotter (http://kmplot.com/analysis/). This online tool was based on data of 1,809 patients downloaded from GEO (Affymetrix HGU133A and HGU133+2 microarrays) (14). The low and high expression was divided by median expression. In Kaplan Meier analysis (Fig. 1), the expression level of FOXP2 was significantly correlated to the patients' relapse-free survival (RFS, $\mathrm{P}=0.0047$, HR=0.79; Fig. 1B), but not to overall survival (OS, $\mathrm{P}=0.18$, Fig. 1A). Among patients with different molecular subtypes, low FOXP2 expression only predicted higher risk of relapse among luminal $\mathrm{A}(\mathrm{P}=0.0037)$ and basal-like breast cancer patients $(\mathrm{P}=0.014)$. The results suggested that low expression of FOXP2 may be associated with recurrence and metastasis in some breast cancer patients.

FOXP2 expression was downregulated in breast cancer compared to normal breast tissue. According to the data from oncomine (www.oncomine.org), the expression of FOXP2 was significantly lower in breast cancer than normal breast tissue $(\mathrm{P}<0.001$; Fig. 2A). The results remained same both in invasive ductal carcinoma and invasive lobular carcinoma (Fig. 2B and C). However, there was no significant difference among patients with and without metastasis (Fig. 2D). We also evaluate the expression level of FOXP2 in different breast cancer cell lines (Fig. 2E and F) as well as 39 patients' samples (Fig. 2G). Interestingly, FOXP2 was significantly less expressed in breast cancer tissue compared to adjacent control tissue $(\mathrm{P}=0.0005)$. The results were consistent with the data from the public database. Table I summarized the clinicopathological characteristics of the patients. In this cohort, the FOXP2 expression was not significantly associated with these clinical features except age.

In addition, we also investigated the frequency of FOXP2 alterations, including amplification and mutations, in breast cancer (Fig. 2H) from cbioprotal (www.cbioportal.org) (15). We found that among 4,786 invasive breast cancer cases from 9 studies, 144 patients had amplification (3\%) and $17(0.4 \%)$ had mutation of this gene.

FOXP2 inhibited breast cancer cells migration and invasion. To validate the function of FOXP2 in breast cancer metastasis and proliferation, we knocked down FOXP2 in MDA-MB-231 and overexpressed it in MDA-MB-231BO and MDA-MB-231HM breast cancer cells via lentivirus infection. The efficiency of infection was confirmed by western blot analysis (Fig. 3A). We then found that the depletion of FOXP2 
A

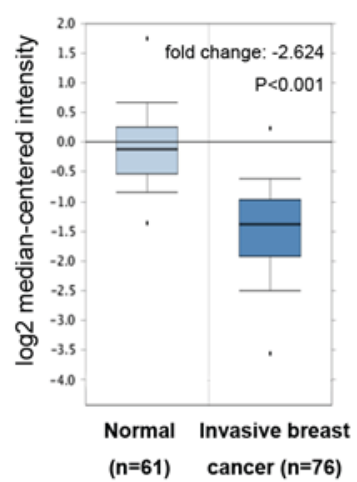

E

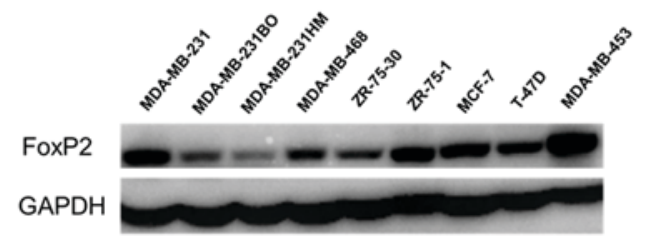

G

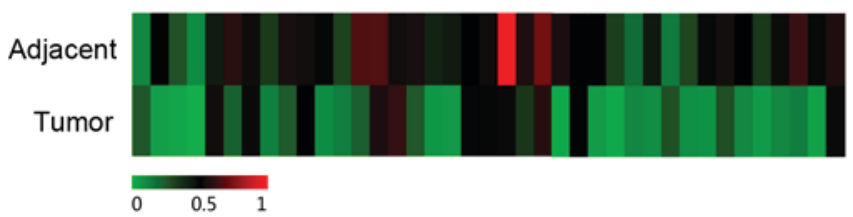

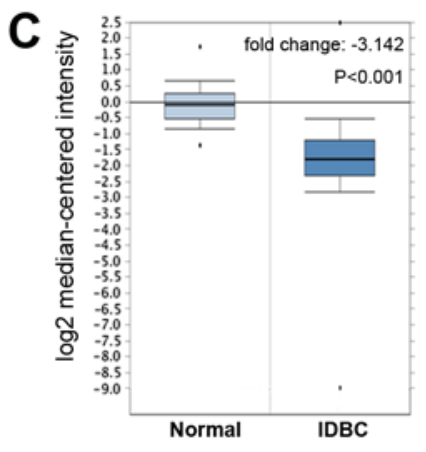
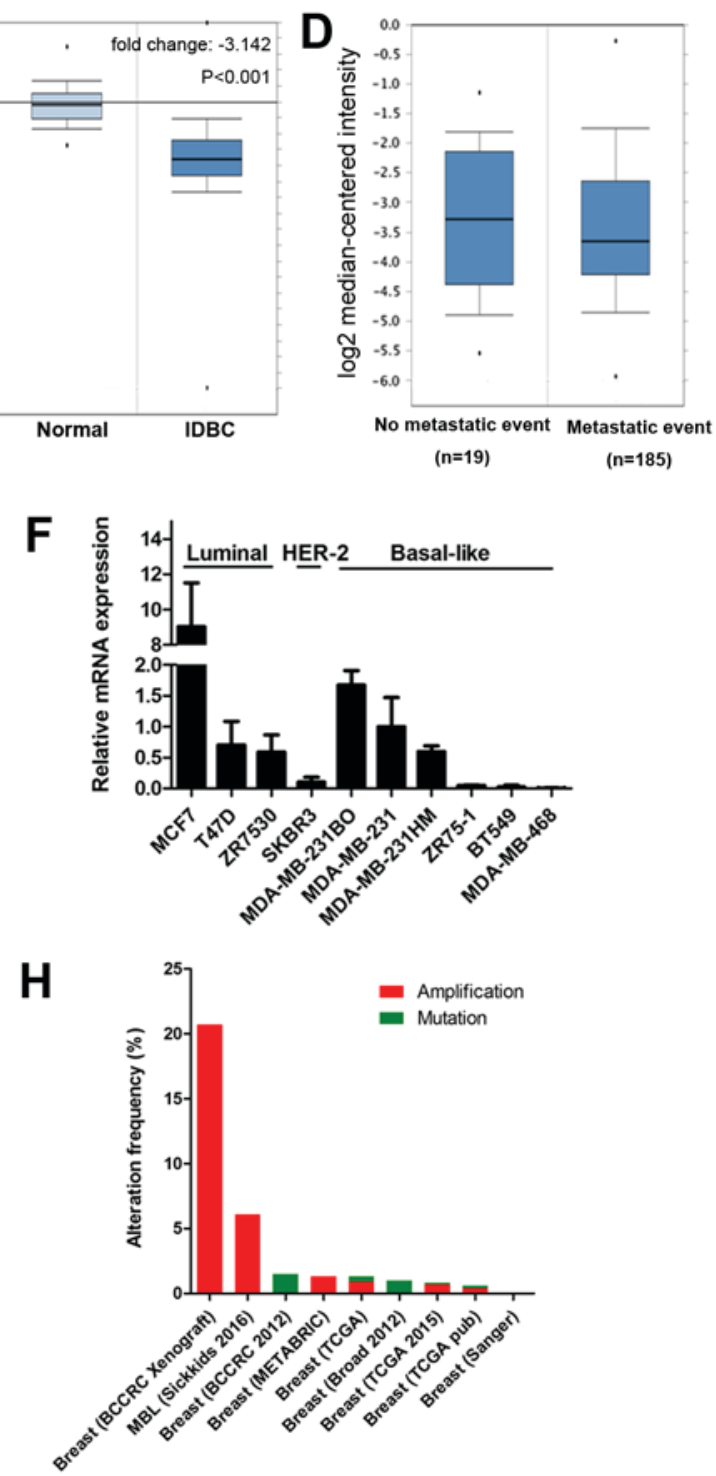

Figure 2. FOXP2 expression is downregulated in breast cancer compared with normal breast tissue. (A-D) Oncomine box plots of FOXP2 expression in TCGA and Bos breast cancer study (oncomine.org) comparing (A) invasive breast cancer (B) ILBC and (C) IDBC with normal breast tissue. (D) No significant differences were observed among patients with and without metastasis. (E) Protein expression level of FOXP2 in different cell lines. (F) Expression level of FOXP2 mRNA in different cell lines. Data were expressed as the mean \pm standard deviation $(\mathrm{n}=3)$. (G) Relative mRNA expression of FOXP2 in 39 breast cancer patients and paired adjacent control tissue. (H) Alteration frequency of FOXP2 gene from cbioportal. ILBC, invasive lobular breast cancer; IDBC, invasive ductal breast cancer.

did not have significant effects on cell proliferation (Fig. 3B). But the wound-healing assay and Transwell assay showed that the knockdown of FOXP2 could promote cell migration and invasion in vitro ( $\mathrm{P}=0.001$; Fig. $3 \mathrm{C}$ and $\mathrm{D})$. Meanwhile, the cell migration and invasion was inhibited after the overexpression of FOXP2 $(\mathrm{P}<0.001)$. Taken together, the results suggested that FOXP2 may play a certain role in breast cancer as a tumor suppressor gene.

Suppression of FOXP2 induced EMT and activated $T G F \beta / S M A D$ signaling pathway. Epithelial-mesenchymal transition (EMT) was known as one of crucial mechanisms in cancer progression and metastasis (16). In order to further investigate the mechanisms of FOXP2 inhibiting breast cancer metastasis, we detected the expression level of several EMT-associated biomarkers in MDA-MB-231 and BT549
(Fig. 4A). The results revealed that suppression of FOXP2 promoted the expression of vimentin and fibronectin in MDA-MB-231. In BT549 cell line, fibronectin and N-cadherin was inhibited after overexpression of FOXP2. The results suggested that FOXP2 could inhibit EMT in breast cancer in vitro.

The activation of TGF $/$ SMAD signaling was known as one of the critical pathways in EMT process and cancer metastasis $(17,18)$. Therefore, we also detected the expression of some important proteins in TGF $\beta$ signaling (Fig. 4B). We found that upregulation of FOXP2 in BT549 could markedly decrease the phosphorylation of SMAD3, enhanced the expression of SMAD4, TGFßR1, ZEB1 and Snail. Meanwhile, suppression of the gene in MDA-MB-231 could cause the reverse effects except ZEB1. The expression of Twist and p-SMAD2 did not seem to show significant differences in the cell lines in 
Table I. Correlation between FOXP2 expression in patients with breast cancer and their clinicopathologic characteristics.

\begin{tabular}{|c|c|c|c|c|c|}
\hline Characteristic & Group & Low expression & High expression & Total & P-value \\
\hline \multirow[t]{2}{*}{ Age } & $\leq 50$ & 12 & 4 & 16 & \multirow[t]{2}{*}{$0.006^{\mathrm{a}}$} \\
\hline & $>50$ & 7 & 16 & 23 & \\
\hline \multirow[t]{3}{*}{ Tumor size $(\mathrm{cm})$} & $\leq 2$ & 5 & 11 & 16 & \multirow[t]{3}{*}{0.097} \\
\hline & $2<\mathrm{n} \leq 5$ & 12 & 9 & 21 & \\
\hline & $>5$ & 2 & 0 & 2 & \\
\hline \multirow[t]{2}{*}{ Lymph nodes } & Negative & 2 & 1 & 3 & \multirow[t]{2}{*}{0.347} \\
\hline & Positive & 14 & 22 & 36 & \\
\hline \multirow[t]{3}{*}{ Menopausal status } & Premenopausal & 10 & 8 & 18 & \multirow[t]{3}{*}{0.497} \\
\hline & Postmenopausal & 9 & 11 & 20 & \\
\hline & NA & 0 & 1 & 1 & \\
\hline \multirow[t]{3}{*}{ Histological grade } & 1 & 1 & 0 & 1 & \multirow[t]{3}{*}{0.260} \\
\hline & 2 & 10 & 7 & 17 & \\
\hline & 3 & 8 & 13 & 21 & \\
\hline \multirow[t]{2}{*}{ ER } & Negative & 4 & 6 & 10 & \multirow[t]{2}{*}{0.522} \\
\hline & Positive & 15 & 14 & 29 & \\
\hline \multirow[t]{2}{*}{ PR } & Negative & 6 & 11 & 17 & \multirow[t]{2}{*}{0.140} \\
\hline & Positive & 13 & 9 & 22 & \\
\hline \multirow[t]{2}{*}{ HER-2 } & Negative & 8 & 9 & 17 & \multirow[t]{2}{*}{0.855} \\
\hline & Positive & 11 & 11 & 22 & \\
\hline
\end{tabular}

${ }^{\mathrm{a}} \mathrm{P}<0.05$. The low and high expression of FOXP2 was divided at the median expression. NA, not applicable; ER, estrogen receptor; PR, progesterone receptor; HER2, human epidermal growth factor receptor-2.
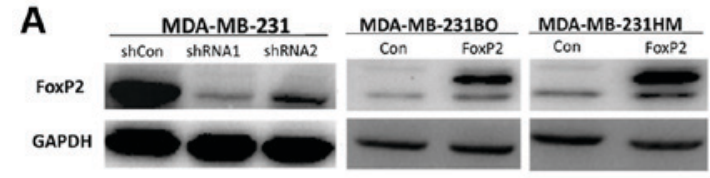

B
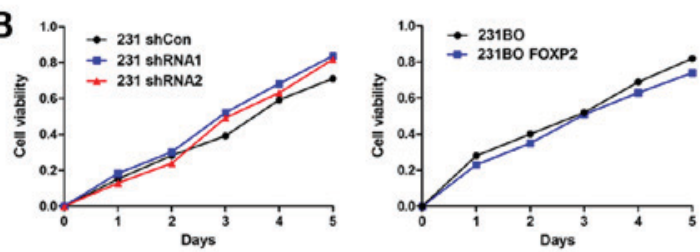

D
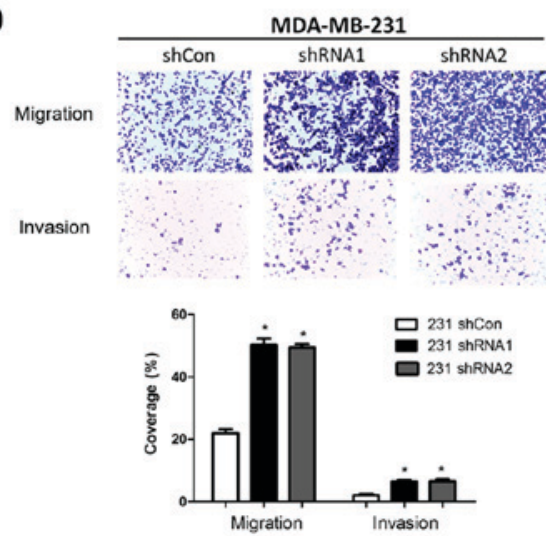

\section{C}
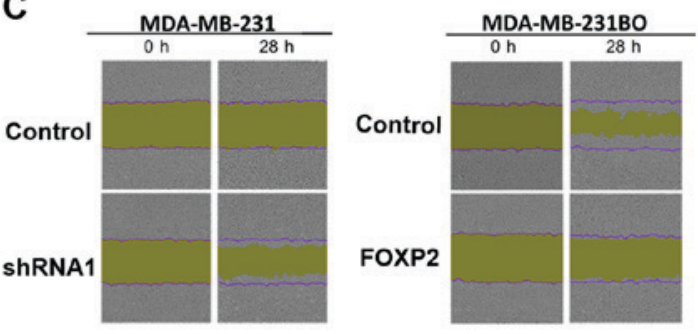

Figure 3. FOXP2 inhibits breast cancer cell migration and invasion in vitro. (A) FOXP2 was knocked down by lentivirus in MDA-MB-231 cells using two independent shRNA constructs and it was constitutively overexpressed in the highly metastatic MDA-MB-231BO and MDA-MB-231HM cells. (B) Proliferation assays did not show significant differences after knockdown or overexpression of FOXP2. (C) Effects of FXOP2 on migration of MDA-MB-231 and MDA-MB-231BO cells in wound-healing assay. Magnification, x100. (D) The migration and invasion Transwell assay of each cell line in vitro. Magnification, $\mathrm{x} 400$. The statistical analysis was performed using student's t-test $(\mathrm{n}=3)$. The error bars represent the standard deviation. ${ }^{*} \mathrm{P}<0.05 \mathrm{vs}$. the control. Sh, short hairpin; Con, control. 
A

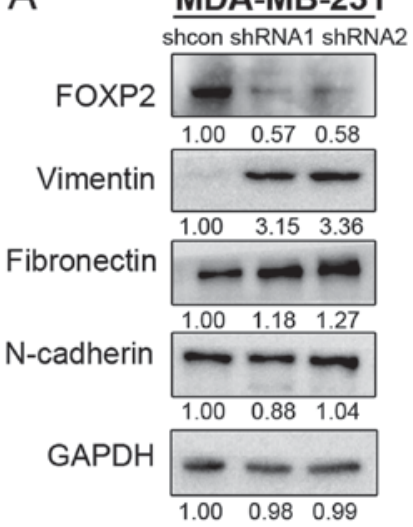

B
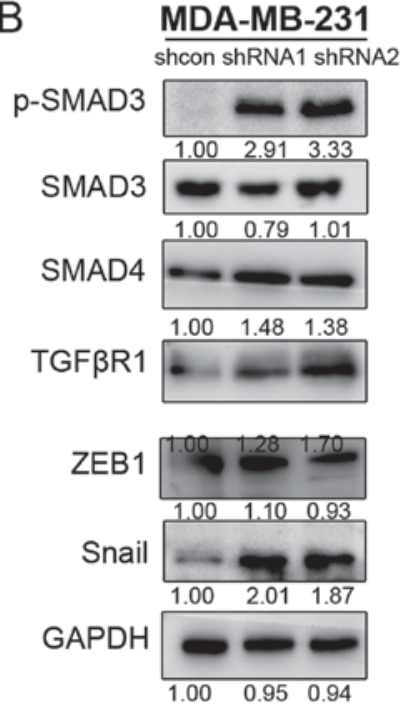
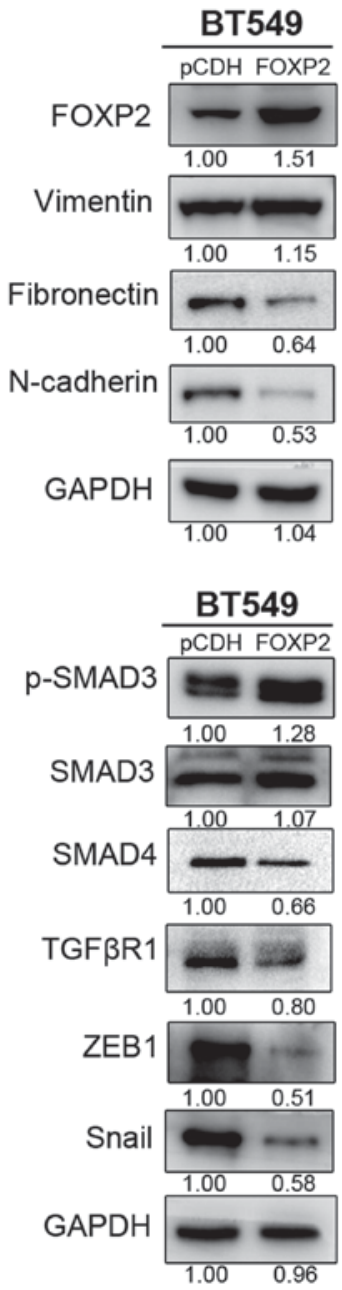

Figure 4. Inhibition of FOXP2 induced EMT and activates the TGF/SMAD signaling pathway. Immunoblotting analysis of mesenchymal markers (vimentin, Fibronectin and N-cadherin) and TGF $3 /$ SMAD signaling proteins (p-SMAD3, SMAD4, TGFßR1, ZEB1, Snail) in (A) MDA-MD-231 and (B) BT549 cell lines. Results are representative of 3 independent experiments. The numbers below the bands are the relative fold changes of the indicated proteins. TGF, transforming growth factor; EMT, epithelial-mesenchymal transition.

this research. The phenomenon indicated that inhibition of FOXP2 induce EMT process in breast cancer probably via TGF $\beta /$ SMAD signaling pathway.

\section{Discussion}

The mechanisms of breast cancer invasion and metastasis are still not fully understood. In our study, we demonstrated FOXP2 might be a potential target for the prevention and treatment of breast cancer metastasis. The function of FOXP2 was initially identified in neurodevelopmental disorders, especially inherited speech-and-language disorders $(19,20)$. Here, we first reported the role of FOXP2 in inhibiting EMT in breast cancer via TGF $\beta /$ SMAD signaling pathway. Upon FOXP2 depletion, the cell mesenchymal biomarkers decreased which indicated higher potential of cell motility and migration. Interestingly, we found that the TGF $\beta$ pathway related proteins (TGF $\beta$ R1, p-SMAD3, SMAD4, Snail) were obviously elevated after downregulation of FOXP2. However, the detailed mechanisms of FOXP2 regulating
TGF $\beta$ pathway are still unclear. TGF $\beta /$ SMAD signaling is a well-known pathway activated during cancer metastasis and transmits signals from cell surface receptors to nuclear transcription (18). Tumor cells usually produce abundant amount of TGF $\beta$ which activates downstream SMAD2/3/4. Then the activated SMADs translocate to nucleus and activated EMT-related transcription factors including Snail, ZEB1/2, Twist, (21). But effective drugs targeting this pathway are still limited clinically, partially because of the side effects (22).

There have been only a few studies investigating the role of FOXP2 in cancer so far. Some studies suggested FOXP2 mediated the crosstalk between breast cancer cells and tumor-associated mesenchymal stem cells (MSCs). For example, Cuiffo et al $(23,24)$ reported that the expression of FOXP2 was regulated by a series of miRNA including miR-199a. They also found that FOXP2 could inhibited breast cancer initiation and colonization through inhibiting a series of cancer stem cell associated factors including c-Myc, Oct-4, CD44, etc. FOXP2 could also be regulated by miR-190 in gastric cancer (9). Upregulation of miR-190 can cause the downregulation of FOXP2 protein expression and then lead to gastric cancer cells migration and invasion. Another study (10) observed transient overexpression of FOXP2 in pre-osteoblast mesenchymal cells influenced a p21-dependent growth arrest checkpoint, which played important roles in the growth of osteosarcoma. These results all confirmed the function of FOXP2 in tumor suppression.

There were also several limitations in our study. For example, the targeted genes regulated by FOXP2 in breast cancer was still unclear. As a transcription factor, FOXP2 need to combine the promoters of the targeted genes to regulate the downstream pathway. But we failed to find the promoters directly targeted by FOXP2. Moreover, we only involved a limited number of patients, most of which had lymph nodes invasion. We did not establish ideal tumour metastatic models in this study. Therefore, the results still need further validation in larger number of cohorts and in vivo.

In conclusion, we identified FOXP2 as a novel tumor suppressor gene in human breast cancer using clinical correlation analysis and in vitro functional metastasis assays. Drugs might be developed to mimic the functions of FOXP2 as a tumor suppressor to prevent or treat breast cancer metastasis in the future. We hope our findings could contribute to the diagnosis and treatment of breast cancer to some extent. Further studies still need to be done in order to investigate the underlying mechanisms of the functions of FOXP2 in the future.

\section{Acknowledgements}

Not applicable.

\section{Funding}

This study was supported by the grant from National Natural Science Foundation of China (no. 81472669) and the Hundred Talents Program of the Health System in Shanghai (no. 2017BR028). 


\section{Availability of data and materials}

The datasets used and/or analyzed during the current study are available from the corresponding author on reasonable request.

\section{Authors' contributions}

WJ, HFS and MTC conceived and designed the study. MTC, LDL and SPG conducted the in vitro experiments. LDL and YZ constructed the plasmids. LPY helped with the tissue preparation and statistical analysis. MTC analyzed the results and wrote the main manuscript. All of the authors reviewed the manuscript.

\section{Ethics approval and consent to participate}

The study was approved by the Ethical Committee and Institutional Review Board of Fudan University Shanghai Cancer Centre and all participants provided written informed consent.

\section{Consent for publication}

Written informed consent was obtained from all participants for the publication of their data.

\section{Competing interests}

The authors declare that they have no competing interests.

\section{References}

1. Siegel RL, Miller KD and Jemal A: Cancer Statistics, 2017. CA Cancer J Clin 67: 7-30, 2017.

2. Gao SP, Sun HF, Jiang HL, Li LD, Hu X, Xu XE and Jin W: Loss of COX5B inhibits proliferation and promotes senescence via mitochondrial dysfunction in breast cancer. Oncotarget 6 : 43363-43374, 2015.

3. Li LD, Sun HF, Liu XX, Gao SP, Jiang HL, Hu X and Jin W: Down-regulation of NDUFB9 promotes breast cancer cell proliferation, metastasis by mediating mitochondrial metabolism. PLoS One 10: e0144441, 2015.

4. Jiang HL, Sun HF, Gao SP, Li LD, Huang S, Hu X, Liu S, Wu J, Shao ZM and Jin W: SSBP1 suppresses TGF $\beta$-driven epithelial-to-mesenchymal transition and metastasis in triple-negative breast cancer by regulating mitochondrial retrograde signaling. Cancer Res 76: 952-964, 2016.

5. Chiu YC, Li MY, Liu YH, Ding JY, Yu JY and Wang TW: Foxp2 regulates neuronal differentiation and neuronal subtype specification. Dev Neurobiol 74: 723-738, 2014.

6. Tsui D, Vessey JP, Tomita H, Kaplan DR and Miller FD: FoxP2 regulates neurogenesis during embryonic cortical development. J Neurosci 33: 244-258, 2013.

7. Fisher SE and Scharff C: FOXP2 as a molecular window into speech and language. Trends Genet 25: 166-177, 2009.
8. Ostrow AZ, Kalhor R, Gan Y, Villwock SK, Linke C, Barberis M, Chen L and Aparicio OM: Conserved forkhead dimerization motif controls DNA replication timing and spatial organization of chromosomes in S. cerevisiae. Proc Natl Acad Sci USA 114: E2411-E2419, 2017.

9. Jia WZ, Yu T, An Q, Yang H, Zhang Z, Liu X and Xiao G: MicroRNA-190 regulates FOXP2 genes in human gastric cancer. Onco Targets Ther 9: 3643-3651, 2016.

10. Gascoyne DM, Spearman H, Lyne L, Puliyadi R, Perez-Alcantara M, Coulton L, Fisher SE, Croucher PI and Banham AH: The forkhead transcription factor FOXP2 is required for regulation of p21WAF1/CIP1 in 143B osteosarcoma cell growth arrest. PLoS One 10: e0128513, 2015.

11. Yan X, Zhou H, Zhang T, Xu P, Zhang S, Huang W, Yang L, Gu X, Ni R and Zhang T: Downregulation of FOXP2 promoter human hepatocellular carcinoma cell invasion. Tumour Biol 36: 9611-9619, 2015.

12. Livak KJ and Schmittgen TD: Analysis of relative gene expression data using real-time quantitative PCR and the 2(-Delta Delta C(T)) method. Methods 25: 402-408, 2001.

13. Jiang HL, Sun HF, Gao SP, Li LD, Hu X, Wu J and Jin W: Loss of RAB1B promotes triple-negative breast cancer metastasis by activating TGF- $\beta /$ SMAD signaling. Oncotarget 6: 16352-16365, 2015.

14. Györffy B, Lanczky A, Eklund AC, Denkert C, Budczies J, Li Q and Szallasi Z: An online survival analysis tool to rapidly assess the effect of 22,277 genes on breast cancer prognosis using microarray data of 1,809 patients. Breast Cancer Res Treat 123: 725-731, 2010.

15. Gao J, Aksoy BA, Dogrusoz U, Dresdner G, Gross B, Sumer SO, Sun Y, Jacobsen A, Sinha R, Larsson E, et al: Integrative analysis of complex cancer genomics and clinical profiles using the cBioPortal. Sci Signal 6: pl1, 2013.

16. Ye X, Brabletz T, Kang Y, Longmore GD, Nieto MA, Stanger BZ, Yang $\mathbf{J}$ and Weinberg RA: Upholding a role for EMT in breast cancer metastasis. Nature 547: E1-E3, 2017.

17. Strzyz P: Cancer biology: TGF $\beta$ and EMT as double agents. Nat Rev Mol Cell Biol 17: 202-203, 2016.

18. Gupta S and Maitra A: EMT: Matter of life or death? Cell 164: 840-842, 2016

19. Konopka G, Friedrich T, Davis-Turak J, Winden K, Oldham MC Gao F, Chen L, Wang GZ, Luo R, Preuss TM and Geschwind DH: Human-specific transcriptional networks in the brain. Neuron 75: 601-617, 2012.

20. Newbury DF and Monaco AP: Genetic advances in the study of speech and language disorders. Neuron 68: 309-320, 2010.

21. Moustakas A and Heldin CH: Mechanisms of TGF $\beta$-induced epithelial-mesenchymal transition. J Clin Med 5: pii: E63, 2016.

22. Cohn A, Lahn MM, Williams KE, Cleverly AL, Pitou C, Kadam SK, Farmen MW, Desaiah D, Raju R, Conkling P and Richards D: A phase I dose-escalation study to a predefined dose of a transforming growth factor- $\beta 1$ monoclonal antibody (T $\beta$ M1) in patients with metastatic cancer. Int J Oncol 45: 2221-2231, 2014

23. Cuiffo BG, Campagne A, Bell GW, Lembo A, Orso F, Lien EC, Bhasin MK, Raimo M, Hanson SE, Marusyk A, et al: MSC-regulated microRNAs converge on the transcription factor FOXP2 and promote breast cancer metastasis. Cell Stem Cell 15: 762-774, 2014.

24. Cuiffo BG and Karnoub AE: Silencing FOXP2 in breast cancer cells promotes cancer stem cell traits and metastasis. Mol Cell Oncol 3: e1019022, 2015.

This work is licensed under a Creative Commons Attribution-NonCommercial-NoDerivatives 4.0 International (CC BY-NC-ND 4.0) License. 